\title{
REVIEW
}

\section{Clinical review: Efficacy of antimicrobial- impregnated catheters in external ventricular drainage - a systematic review and meta-analysis}

\author{
Xiang Wang, Yan Dong, Xiang-Qian Qi, Yi-Ming Li, Cheng-Guang Huang* and Li-Jun Hou*
}

\begin{abstract}
To assess the efficacy of antimicrobial-impregnated catheters in preventing catheter-related infections during external ventricular drainage (EVD), we performed a meta-analysis and systematic review. We systematically searched Medline, Embase, and the Cochrane Library. All randomized controlled trials (RCTs) and nonrandomized prospective studies (NPSs) related to antimicrobial-impregnated EVD catheters were included. The primary outcome was the rate of cerebrospinal fluid infection (CFI). The secondary outcomes included the rate of time-dependent $\mathrm{CFI}$ and catheter bacterial colonization. We further performed subgroup analysis, meta-regression analysis, and microbial spectrum analysis. Four RCTs and four NPSs were included. The overall rate of CFIs was 3.6\% in the antimicrobialimpregnated catheter group and $13.7 \%$ in the standard catheter group. The pooled data demonstrated that antimicrobial-impregnated catheters were superior to standard catheters in lowering the rate of CFIs (odds ratio $(O R)=0.25,95 \%$ confidence interval $(C I)=0.12$ to $0.52, P<0.05)$. In survival analysis, the 20 -day infection rate was significantly reduced with the use of antimicrobial-impregnated catheters (hazard ratio $=0.52,95 \% \mathrm{Cl}=0.29$ to 0.95 , $P$ <0.05). Furthermore, a significantly decreased rate of catheter bacterial colonization was noticed for antimicrobialimpregnated catheters $(\mathrm{OR}=0.37,95 \% \mathrm{Cl}=0.21$ to $0.64, P<0.05)$. In subgroup analyses, although significant results remained for RCTs and NPSs, a subgroup difference was revealed $(P<0.05)$. Compared with standard catheters, a significantly lower rate of CFls was noticed for clindamycin/rifampin-impregnated catheters $(\mathrm{OR}=0.27,95 \% \mathrm{Cl}=0.10$ to $0.73, P<0.05)$ and for minocycline/rifampin-impregnated catheters $(\mathrm{OR}=0.11,95 \% \mathrm{Cl}=0.06$ to $0.21, P<0.05)$.

However, no statistical significance was found when compared with silver-impregnated catheters $(O R=0.33,95 \%$ $\mathrm{Cl}=0.07$ to $1.69, P=0.18$ ). In microbial spectrum analysis, antimicrobial-impregnated catheters were shown to have a lower rate of Gram-positive bacterial infection, particularly the coagulase-negative Staphylococcus. In conclusion, the use of antimicrobial-impregnated EVD catheters could be beneficial for the prevention of CFI and catheter bacterial colonization. Although antibiotic-coated catheters seem to be effective, no sufficient evidence supports the efficacy of silver-impregnated catheters.
\end{abstract}

\section{Introduction}

External ventricular drainage (EVD) is widely used in current neurosurgical practice. EVD is indispensable for patients with acute increase of intracranial pressure, intraventricular hemorrhage, and obstructive hydrocephalus. Although it has potential therapeutic effects, a high risk of catheter-related cerebrospinal fluid infection (CFI) still remains unsolved [1]. Apart from leading to a poor outcome, the infection also contributes to increased

*Correspondence: lj_hou@hotmail.com, huang64@163.com

Department of Neurosurgery, Shanghai Institute of Neurosurgery, Military Institute of Neurosurgery, Changzheng Hospital, Second Military Medical University, 415 Fengyang Road, Shanghai, China length of stay in the ICU and hospital and to higher total hospital costs [2,3].

Prophylactic systemic antibiotics (PSA) are routinely administered for patients with EVD in many institutions. However, PSA may not significantly lower the incidence of ventriculitis [4]. The antimicrobial-impregnated catheter has emerged as an alternative strategy. Currently, catheters impregnated with clindamycin/rifampin (C/R), minocycline/rifampin (M/R), and silver are commercially available [5]. Most studies showed benefits of antimicrobial-impregnated EVD catheters in preventing infections [3,6-10]. However, some studies did not demonstrate positive conclusions [11-13]. Gram-positive organisms are predominant in microbiological cultures of cerebrospinal fluid (CSF) samples. Due to the selective pressure 
exerted by pervasive use of prophylactic antibiotics, an increasing rate of Gram-negative infections has been reported $[1,14,15]$. The protective effects of antimicrobial-impregnated catheters against Gram-positive or Gram-negative infections have not been clarified. In light of those pending issues, we performed this systematic review and meta-analysis, aiming to evaluate the efficacy of antimicrobial-impregnated EVD catheters.

\section{Methods}

\section{Search strategy}

Our meta-analysis was conducted in accordance with the Preferred Reporting Items for Systematic Reviews and Meta-analysis (PRISMA) statement [16] (see Additional file 1). We systematically searched Medline (Ovid), Embase, and the Cochrane Library until October 2012, with language restricted to English, and identified all prospective studies related to the use of antimicrobial-impregnated EVD catheters. Our search strategy included terms for antimicrobial catheter (antibiotic-impregnated/ coated catheter that includes minocycline/rifampin catheters and clindamycin/rifampin catheters, and silverimpregnated/coated catheter), procedures (external ventricular drainage, ventriculostomy, and shunt), and study design (randomized controlled trials, prospective studies). Furthermore, we manually searched the references of identified papers to find additional eligible studies.

\section{Selection criteria}

Studies were included into the meta-analysis if they: were randomized controlled trials (RCTs) or nonrandomized prospective studies (NPSs) of patients with antimicrobialimpregnated catheters; compared the antimicrobial catheter (treatment arm) with the standard catheter (control arm) in the prevention of catheter-related infections in EVD (we eased the criteria for the control arm and allowed the use of well-matched historical controls); reported original data; and reported a risk estimate (that is, odds ratio (OR), relative risk, or hazard ratio (HR)) for the utilization of antimicrobial-impregnated catheters to subsequent rate of catheter-related infections. We referred to the Centers for Disease Control/National Healthcare Safety Network definition of ventriculitis to identify the infectious outcome. According to the criteria, clinical manifestations are emphasized besides laboratory tests [17]. However, most studies had no defined requirements for clinical manifestations. We therefore modified the aforementioned criteria and viewed CFI as the primary outcome, defined as positive CSF culture or staining, or a significant increase of CSF white cell count, with or without clinical manifestations [17]. The rate of time-dependent CFI and catheter bacterial colonization were explored as secondary outcomes. Catheter bacterial colonization was defined as positive culture of explanted catheters in vitro. For multiple reports on the same study, the one with complete information was selected for meta-analysis.

\section{Data extraction and quality assessment}

Two assessors (XW and YD) independently reviewed the full manuscripts of eligible studies. Data were extracted in standardized data-collection forms. The extracted information included the following items: first author's name; year of publication; sample size; population; gender; treatment arms; usage of PSA; duration of catheter placement; and catheter-related infection outcomes (infection rate, organism analyses). Any disagreement was resolved by discussion or by consulting a senior scholar (C-GH). Selected RCTs were critically appraised using the Jadad scale (randomization, 2 points; blinding, 2 points; and attrition information, 1 point) [18]. The Newcastle-Ottawa scale was used to evaluate the methodological quality of prospective cohort studies [19]. The quality of a study was judged by the selection of the study groups, the comparability of the groups, and the ascertainment of the outcome.

\section{Statistical analysis}

Review Manager 5.1.7 (Cochrane Collaboration, Copenhagen, Denmark) was used to perform the meta-analysis. At event rates $<1 \%$, Peto's OR is suggested to be the least biased and most powerful method [20]. However, Peto's OR is less appropriate when the event rates are $>5 \%$, the treatment effects are significant, and the number of treated and control participants is imbalanced [20]. We therefore used different statistical methods. Besides calculating the Peto OR with a fixed-effect model, we calculated the OR with the Mantel-Haenszel method and the random-effects model $[21,22]$. When the event rates were $<1 \%$, the treatment effects were small to moderate, and the number in each group was balanced. The Peto OR was thus chosen as the primary statistical method. Otherwise, the OR was used instead.

The comparison of different meta-analytic methods was included into the sensitivity analyses. Additionally, sensitivity analysis was conducted by excluding the individual studies one by one.

Considering the infection events as time-dependent data, the HR was employed for analysis [23]. If available, HRs and associated variances were extracted directly. Otherwise they were estimated indirectly from other summary statistics (95\% confidence intervals (CIs), $P$ values, total number of events) or from data from published Kaplan-Meier curves [23]. The Kaplan-Meier curve was read with the Engauge Digitizer version 2.15 free software [24]. The log HR and its standard error were calculated and further pooled by Review Manager 5.1.7 with the random-effects model. 
The $I^{2}$ statistic was used to reveal the heterogeneity of treatment effects. $I^{2}$ of 0 to $40 \%$ indicates unimportant heterogeneity, 30 to $60 \%$ indicates moderate heterogeneity, 50 to $90 \%$ indicates substantial heterogeneity, and 75 to $100 \%$ indicates considerable heterogeneity [25]. Heterogeneity was further explored by subgroup analyses and meta-regression. Four potential sources of heterogeneity were analyzed: study design (RCT or NPS); catheter type $(C / R, M / R$, or silver); sample size; and publication year. Meta-regression analysis was conducted by Stata 12.0 (Stata Corporation, College Station, TX, USA) with the random-effects model to assess whether a specific covariate influenced the effect. The data were expressed with $95 \%$ CIs. Two-tailed $P<0.05$ was deemed statistically significant. The publication bias was examined by the funnel plots on Review Manager 5.1.7, and statistically by Egger's regression model, calculated by Stata 12.0.

\section{Results}

We identified 144 relevant articles from the initial search and 115 were excluded after a preliminary review. The remaining 29 studies were retrieved for detailed assessment. Sixteen retrospective cohort studies and four studies of ventriculoperitoneal shunt were excluded, with five RCTs and four NPSs related to EVD remaining. Further, one RCT was discarded because no infection event was observed in both the intervention and control groups [11]. Excluding the zero-total-event trials from meta-analysis is common practice because they do not contribute to treatment effects analysis [26]. The flow diagram is shown in Figure 1. Eight studies were included in the meta-analysis [6-10,12,13,27]. Their characteristics are presented in Table 1 . Three NPSs prospectively collected the antimicrobial-impregnated catheter data, and selected well-matched historical controls [8-10]. The sample size ranged from 39 to 1,634. Most studies involved an adult population and used PSA. The quality of RCTs and NPSs were moderately satisfactory.

\section{Infection rate}

All studies reported the overall rate of CFI in each arm. Accordingly, the OR of the CFI rate could be obtained. Although the definition of infection outcomes varied across studies (Table 1), they were in accordance with our predefined criteria. As a considerable number of patients underwent EVD several times, we considered the CFI rate to be EVD-frequency related. We thus counted 1,875 EVD events with antimicrobial-impregnated catheters and 1,116 control EVD events with standard catheters. The overall rates of CFIs were $3.6 \%$ in patients with antimicrobial-impregnated catheters and $13.7 \%$ in patients with standard catheters. The pooled data demonstrated that antimicrobial-impregnated catheters were significantly superior to standard catheters in lowering the CFI rate $(\mathrm{OR}=0.25,95 \% \mathrm{CI}=0.12$ to $0.52, P<0.05$; Figure 2$)$. As for the Peto OR, the overall difference remained significant $(\mathrm{OR}=0.32,95 \% \mathrm{CI}=0.24$ to $0.42, P<0.05)$. When sequentially excluding studies from the main pooled analysis, the results were not significantly affected.

Subgroup analyses were warranted in light of the substantial heterogeneity across studies $\left(I^{2}=75 \%\right)$. We analyzed RCTs and NPSs to ascertain the potential bias resulting from study design. Pooled data of four RCTs showed a significant protective effect against CFIs for antimicrobial-impregnated catheters $(\mathrm{OR}=0.49,95 \%$ $\mathrm{CI}=0.27$ to $\left.0.89, P<0.05, I^{2}=46 \%\right)$. A significant result was also demonstrated in analysis of four NPSs (OR = $0.12,95 \% \mathrm{CI}=0.07$ to $0.21, P<0.01, I^{2}=0$ ) (Figure 2).

Of the eight studies, one RCT and one NPS assessed the effect of silver-impregnated catheters on CFI prevention. As shown in Figure 3, less patients with silverimpregnated catheters developed CFIs (10.8\%) compared with standard catheters (21.9\%), without statistical significance $(\mathrm{OR}=0.33,95 \% \mathrm{CI}=0.07$ to $1.69, P=0.18)$. Harrop and colleagues prospectively utilized $C / R$ and $\mathrm{M} / \mathrm{R}$ catheters in different observational periods and thus they were pooled separately [27]. Overall, five studies examined the effect of C/R-impregnated catheters and showed a significant association with a lower rate of CFIs $(\mathrm{OR}=0.27,95 \% \mathrm{CI}=0.10$ to $0.73, P<0.05)$. Two studies evaluated the effect of $M / R$-impregnated catheters, indicating a significant correlation with a lower rate of CFIs $(\mathrm{OR}=0.11,95 \% \mathrm{CI}=0.06$ to $0.21 ; P<0.05)$ (Figure 3). When sensitivity analyses were performed with Peto ORs, the only significant change was found for silver-impregnated catheters (Peto $\mathrm{OR}=0.45,95 \% \mathrm{CI}=$ 0.25 to $0.81, P<0.05)$.

To examine the extent to which differences in the infection rate could be explained, the study design, publication year, and sample size were considered as independent variables in meta-regression analyses. A significant independent effect for study design was indicated $(P=$ 0.018). However, neither the publication year nor the sample size had an independent effect on the CFI outcome $(P=0.103$ and $P=0.399$, respectively) (Figure 4$)$.

\section{Estimation of the time-dependent infection rate}

The EVD catheter was usually temporarily implanted for a short period. Theoretically, all EVD catheters may become infected given enough time. In consideration of the time to infection or censoring, a time-dependent infection rate was explored. Four RCTs illustrated a Kaplan-Meier curve that demonstrated EVD without infection $[6,7,12,13]$. The infection rate reached a steady level after day 20 following catheter implantation in all studies, and thus the 20-day infection rate was investigated. Three studies presented the $P$ value of the 


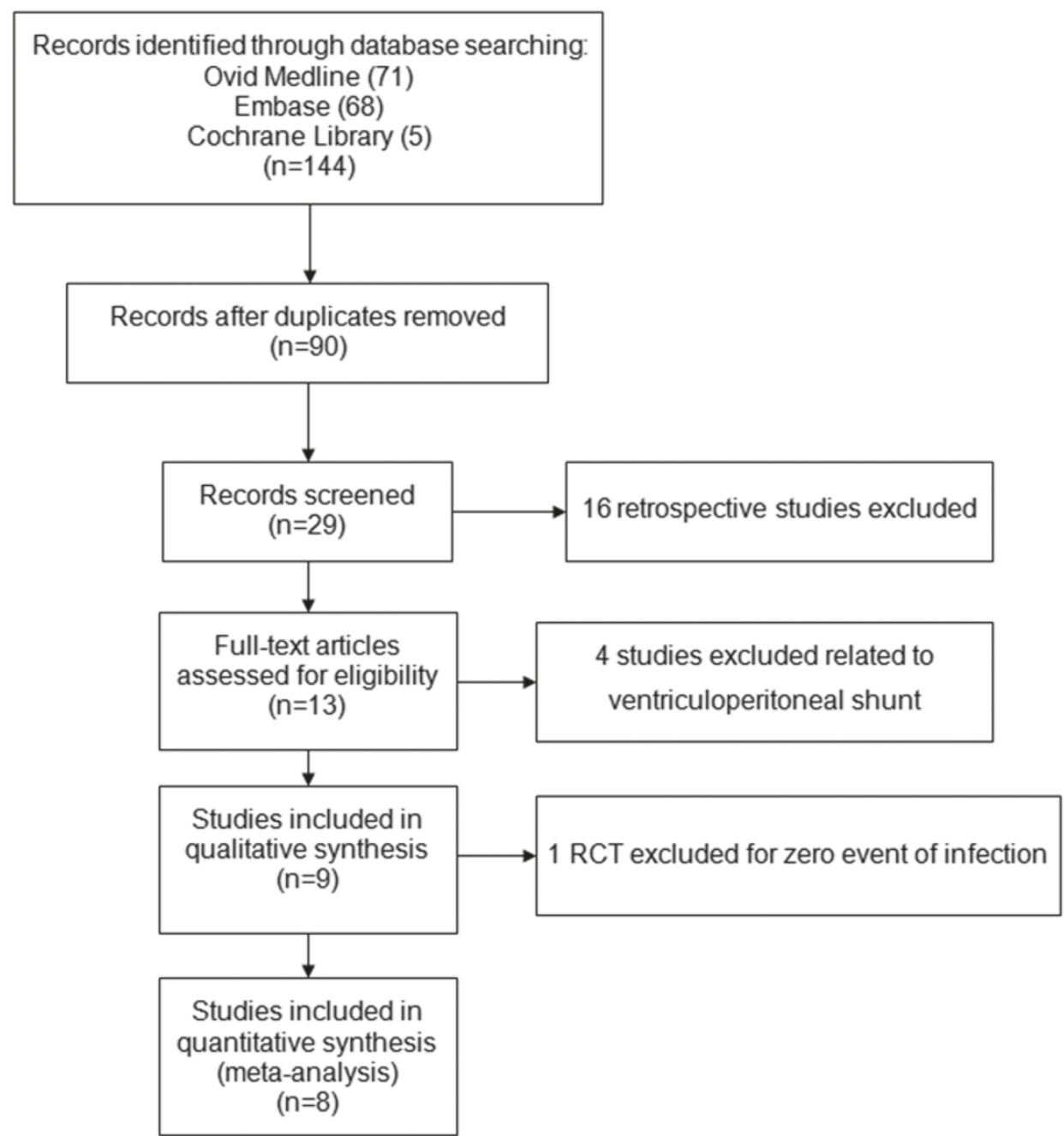

Figure 1. Selection of studies for our meta-analysis. RCT, randomized controlled trial.

log-rank test $[6,12,13]$, whereas the SILVER trial solely presented the Kaplan-Meier curve [7]. The estimated log HR and the standard error of log HR were indirectly calculated, and the pooled results demonstrated that antimicrobial-impregnated catheters were helpful for prolonging the EVD catheter period without infection $(\mathrm{HR}=0.52,95 \% \mathrm{CI}=0.29$ to $0.95, P=0.03)$ (Figure 5$)$.

\section{Catheter bacterial colonization}

Three studies of $C / R$ catheters reported catheter bacterial colonization $[6,12,13]$. Totally, there were 24 events of bacterial colonization (6.2\%) in 389 antimicrobialimpregnated catheters and 48 events of bacterial colonization (12.5\%) in 384 standard catheters. The pooled results showed that antimicrobial-impregnated catheters were associated with lower risk of catheter bacterial colonization $(\mathrm{OR}=0.37,95 \% \mathrm{CI}=0.21$ to $0.64, P<0.05)$ (Figure 6).

\section{Microbial spectrum analyses}

The specific microbial spectra in CFIs were investigated in five studies (Figure 7). For coagulase-negative Staphylococcus, antimicrobial-impregnated catheters were superior to standard catheters in lowering the infection rate $(\mathrm{OR}=0.17,95 \% \mathrm{CI}=0.06$ to $0.53, P<0.05)$. For Staphylococcus aureus, a trend but no statistical significance to the decrement of infection rate was shown for the antimicrobial-impregnated catheter group $(\mathrm{OR}=$ $0.41,95 \% \mathrm{CI}=0.07$ to $2.33, P>0.05)$. Similarly, for Gramnegative rods there was a trend of decreasing infection rate in the antimicrobial-impregnated catheter group compared with the standard catheter group without statistical significance $(\mathrm{OR}=0.44,95 \% \mathrm{CI}=0.07$ to 2.58 , $P>0.05)$. When analyzing Gram-positive cocci, antimicrobial-impregnated catheters were associated with a lower rate of CFI $(\mathrm{OR}=0.18,95 \% \mathrm{CI}=0.08$ to 0.39 , $P<0.05)$. Sensitivity analysis was performed by 


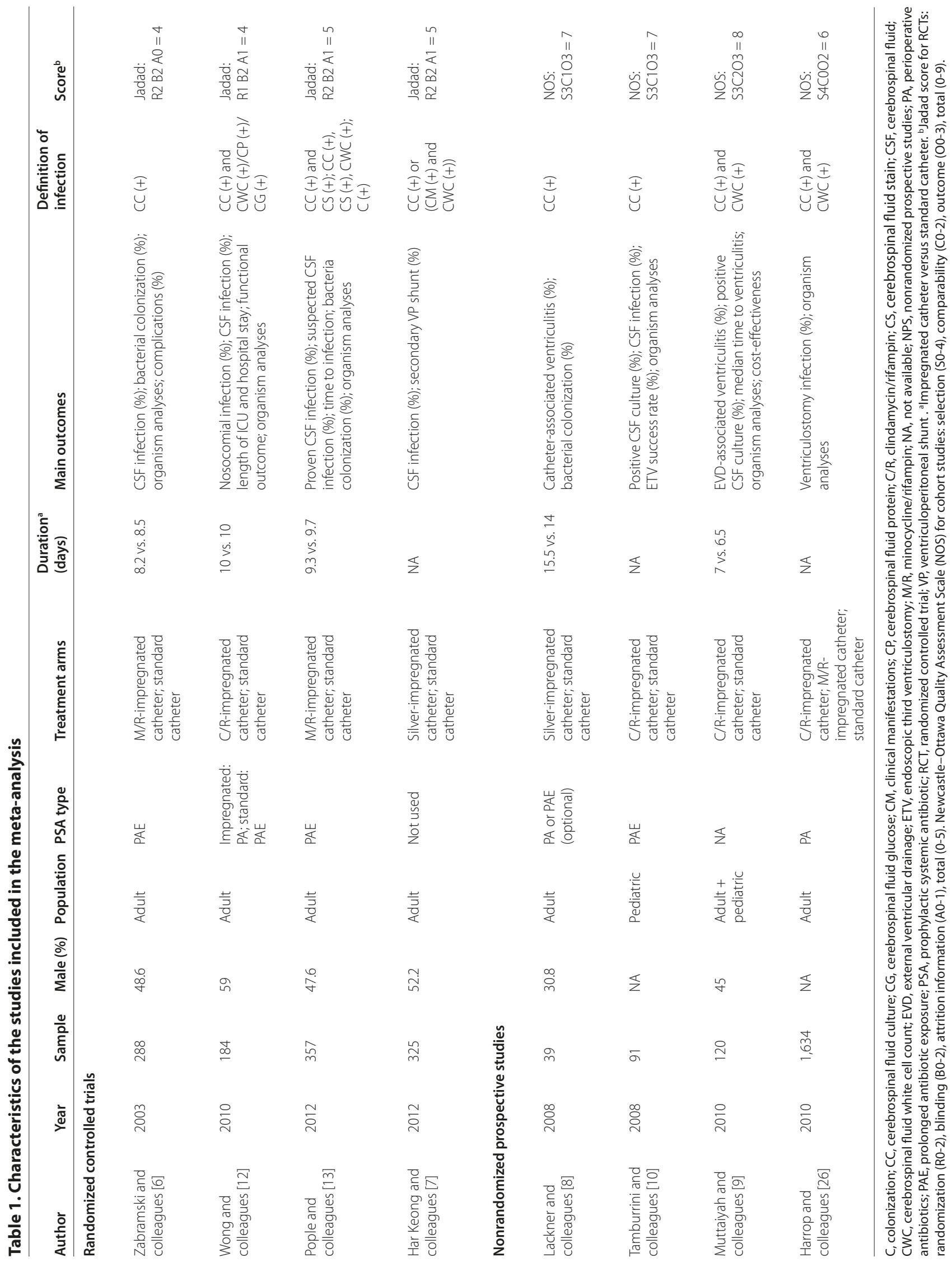




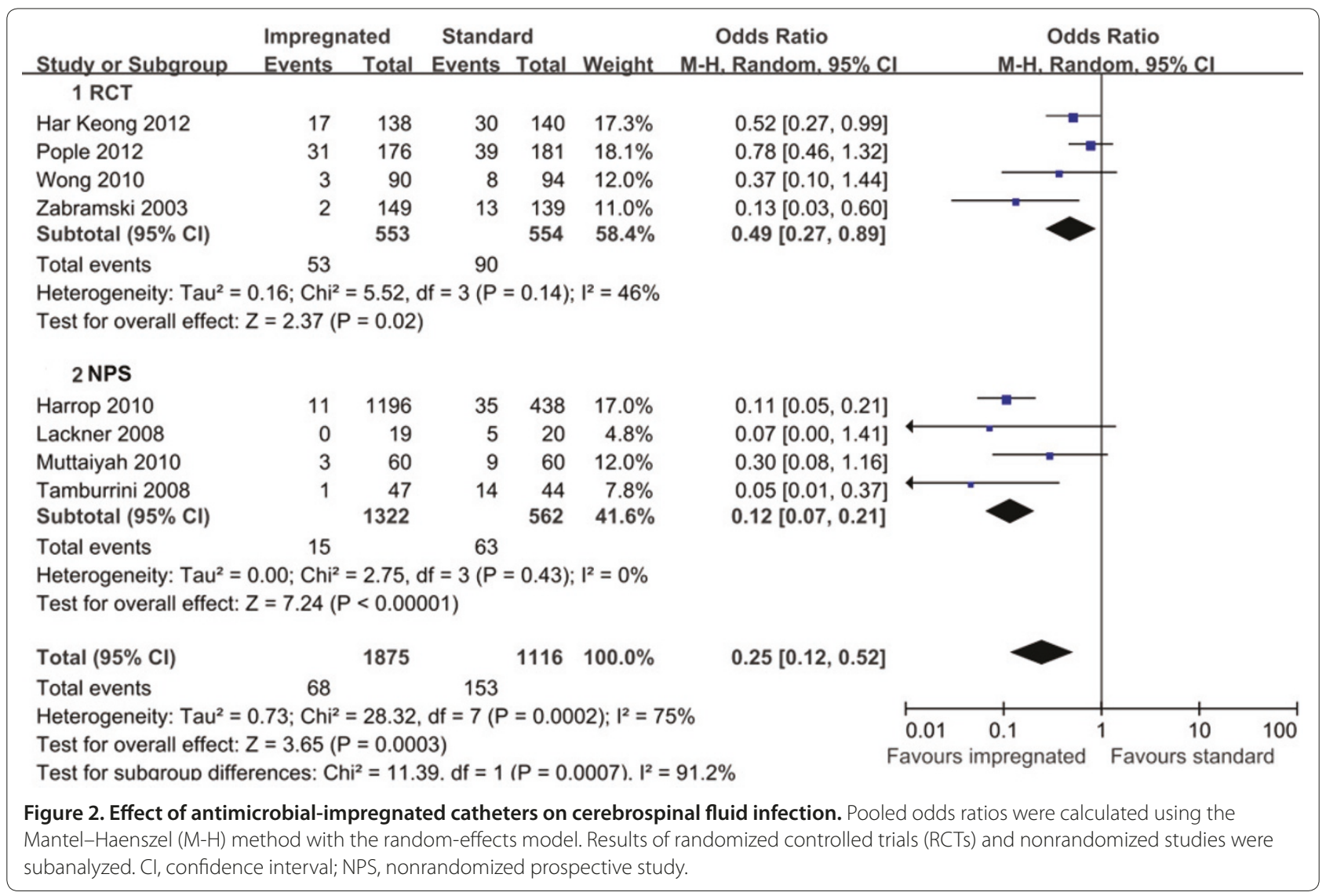

calculating the Peto OR and no significant change was found.

Only few studies were available for resistant organisms, which mainly included drug-resistant $S$. aureus, Acinetobacter, Pseudomonas, and fungus. Zabramski and colleagues isolated rifampin-resistant Pseudomonas species [6]. Wong and colleagues detected one case with methicillinresistant S. aureus infection. They also investigated the resistant organisms in the overall nosocomial infections. Resistant infections occurred in $27 / 90$ patients in the antimicrobial-impregnated catheter group and in 34/94 patients in the control group, with no significant difference [12]. Methicillin-resistant S. aureus was also reported in the silver-impregnated catheter group in the SILVER trial [7].

\section{Evaluation for publication bias}

The funnel plots of all data were found to be symmetrical, suggesting a low likelihood of publication bias (Figure 8). No publication bias was found by Egger's test either $(P=$ $0.178)$.

\section{Discussion}

In our meta-analysis, the pooled Mantel-Haenszel OR demonstrated that antimicrobial-impregnated catheters were associated with a decreased rate of CFIs, which was not significantly altered with the Peto OR. A lower rate of catheter bacterial colonization was also indicated in patients with antimicrobial-impregnated catheters. Furthermore, the pooled HR of the 20-day infection rate indicated that patients with antimicrobial-impregnated catheters had a significantly decreased risk of CFI compared with those with standard catheters. Our research suggested a protective effect associated with the use of antimicrobial-impregnated catheters for CFI prevention.

In meta-regression analyses, neither the publication year nor the sample size had a significant effect on the outcome, whereas the study design was shown to exert an independent effect. Further, in subgroup analyses, although results stratified by study design were statistically significant, a significant subgroup difference was found. Considering that the NPSs contributed $75 \%$ of patients to the meta-analysis, most of the observed signals may be related to NPSs and not to RCTs. Antibiotic-coated catheters were shown to be effective. However, for the silver-coated catheter, our data did not demonstrate benefit. Compared with antibiotic agents, sliver is expected to exhibit a more widespread antibiotic spectrum, defending against all Gram-positive and Gram-negative bacteria and Candida spp. Nevertheless, 


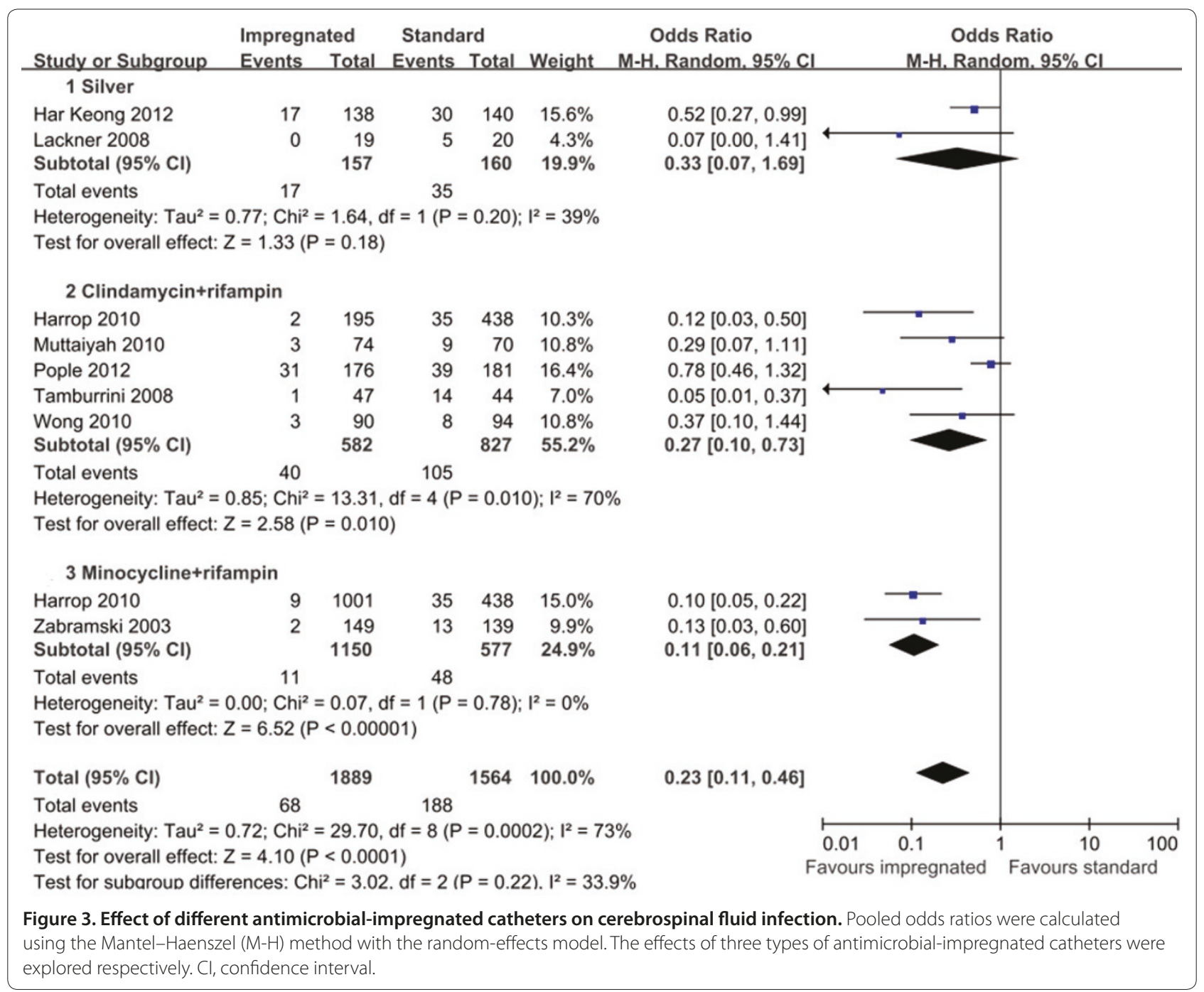

previous clinical experience of the silver central venous line catheter demonstrated that it is inferior to antibioticimpregnated catheters for preventing central venous line infection [28]. The comparison of the silver-impregnated catheter with antibiotic-impregnated catheters therefore calls for further investigation.

CFIs often result from contamination along the tract with normal skin flora, such as Staphylococcus spp. and Streptococcus spp. [29]. Gram-positive cocci comprise the majority of isolates in EVD procedures [30]. Coagulasenegative Staphylococci, predominantly Staphylococcus epidermidis, have been identified as the most frequent causative agent of CFIs [31]. Our results demonstrated that antimicrobial-impregnated catheters might reduce the infection risk of Gram-positive cocci, especially S. aureus. Nevertheless, no statistically favorable results showed their effects on reducing infection with S. epidermidis or Gramnegative rods. Of note, the reason might be that the sample sizes were overall too small to produce conclusive results.

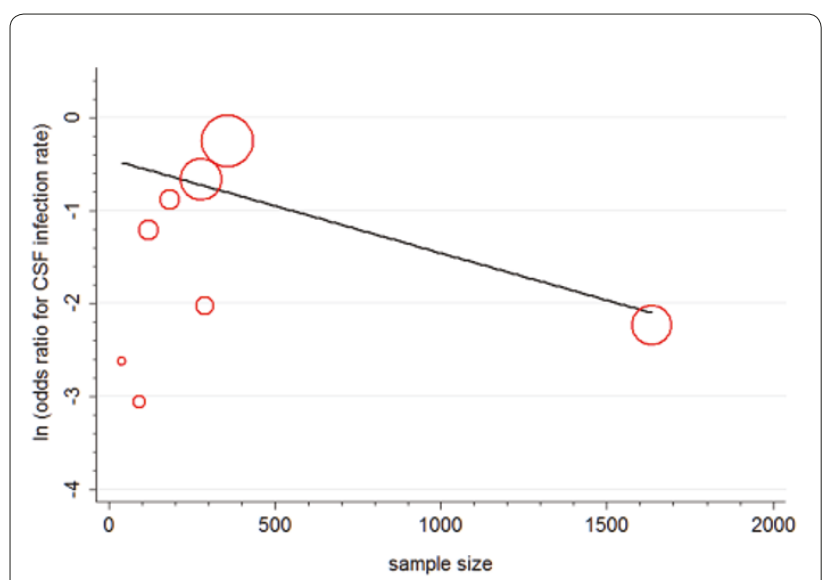

Figure 4. Meta-regression of the log odds ratio for cerebrospinal fluid infection rate against the sample size. Size of circle is proportional in area to the study's weight in the analysis. $P=0.399$. CSF, cerebrospinal fluid. 


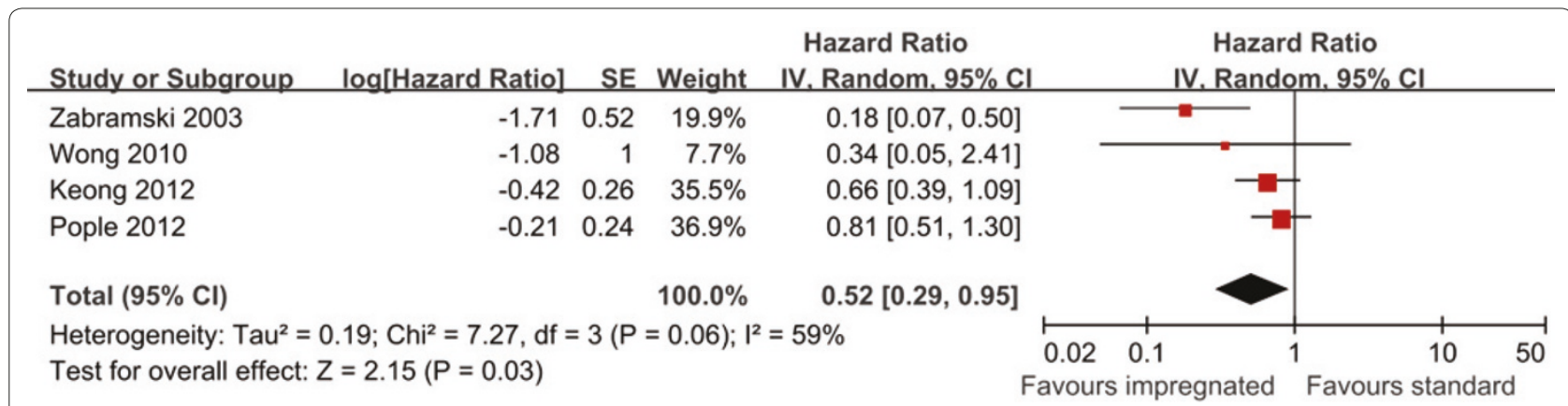

Figure 5. Effect of antimicrobial-impregnated catheters on catheter bacterial colonization. Pooled hazard ratios were estimated using the inverse variance method, with the random-effects model. Cl, confidence interval; SE, standard error.

\begin{tabular}{|c|c|c|c|c|c|c|c|c|c|c|}
\hline Study or Subgroup & \multicolumn{2}{|c|}{ Impregnated } & \multicolumn{2}{|c|}{ Standard } & Weiaht & $\begin{array}{l}\text { Odds Ratio } \\
\text { M-H, Random, } 95 \% \mathrm{Cl}\end{array}$ & & \multicolumn{2}{|c|}{$\begin{array}{c}\text { Odds Ratio } \\
\mathrm{M}-\mathrm{H} \text {, Random, } 95 \% \mathrm{Cl}\end{array}$} & \\
\hline Pople 2012 & 0 & 176 & 3 & 181 & $3.5 \%$ & $0.14[0.01,2.82]$ & & & & \\
\hline Wong 2010 & 2 & 90 & 5 & 94 & $11.2 \%$ & $0.40[0.08,2.14]$ & & & & \\
\hline Zabramski 2003 & 22 & 123 & 40 & 109 & $85.3 \%$ & $0.38[0.21,0.69]$ & & & & \\
\hline Total $(95 \% \mathrm{Cl})$ & & 389 & & 384 & $100.0 \%$ & $0.37[0.21,0.64]$ & & & & \\
\hline Total events & 24 & & 48 & & & & & & & \\
\hline $\begin{array}{l}\text { Heterogeneity: } \mathrm{Tau}^{2}= \\
\text { Test for overall effect: }\end{array}$ & $\begin{array}{l}.00 ; \mathrm{Chi}^{2} \\
=3.53(\mathrm{P}\end{array}$ & $\begin{array}{l}=0.40, \mathrm{c} \\
=0.000\end{array}$ & ff $=2(P=$ & $=0.82)$ & $1^{2}=0 \%$ & & $\begin{array}{l}0.01 \\
\text { avours }\end{array}$ & $\begin{array}{l}0.1 \\
\text { mpregnated }\end{array}$ & $\begin{array}{l}10 \\
\text { Favours stan }\end{array}$ & $\begin{array}{l}100 \\
\text { ard }\end{array}$ \\
\hline
\end{tabular}

Figure 6. Effect of antimicrobial-impregnated catheters on catheter colonization. Pooled odds ratios were calculated using the MantelHaenszel $(\mathrm{M}-\mathrm{H})$ method with the random-effects model. Cl, confidence interval.

Our meta-analysis is different from previous reviews in several aspects. In the earlier Cochrane review, only two RCTs were included without sufficient data to elucidate the efficacy of antimicrobial-impregnated catheters [32]. In another systematic review, Sonabend and colleagues investigated both the effects of prophylactic antibiotics and antibiotic-coated EVD catheters on ventriculostomyrelated infections, and only one RCT of EVD was selected [2]. For another meta-analysis by Thomas and colleagues, the major limitation is the inclusion of considerable lowquality retrospective studies, and mixed assessment of shunt and EVD. Besides, the neonatal population was mainly evaluated [33]. Compared with previous studies, our research consisted of all up-to-date prospective studies of relatively high quality and employed the Mantel-Haenszel OR and the Peto OR to estimate the time-dependent infection rate. Additionally, the benefits of antimicrobial EVD catheters against different microbial spectrums, as well as the efficacy of different catheters, were explored.

We are aware of the limitations for our meta-analysis. One limitation was the quality of studies. Although evidence from RCTs was ideal, only a few RCTs were eligible. Eventually, we identified four RCTs and four NPSs. In three NPSs, historical control groups were employed, which might lead to a failure to identify infections in this group, thus contributing to an underestimated infection rate [8-10]. Although selection bias has been suggested to be reduced by well-matched historical controls $[8-10,34]$, its potential existence should not be neglected. The heterogeneity was found to be moderate to considerable, which might arise from the varied infection definition, inappropriate study design with multiple confounding factors, and too small a sample size. Kubilay and colleagues suggested that the implementation of a ventriculostomy placement bundle, including antimicrobial-impregnated catheters, administration of PSA and a series of sterile techniques, dramatically decreased EVD-related infections [3]. The nonstandard surgical procedures between studies therefore probably served as a potential source of heterogeneity. The underlying diseases and severity of conditions also play roles in determining the infectious outcomes, beyond the impregnated antimicrobial agents. It is implied that the efficacy of antimicrobial EVD catheters might be most pronounced in patients with elevated intracranial pressure etiologies of higher infection risk (head trauma, prior shunt failure) [13]. However, the indication of antimicrobial-impregnated catheters for specific populations still remains to be clarified. Especially, the potential confounders are difficult to balance in NPSs. The rate of CFIs is rather low. It has 


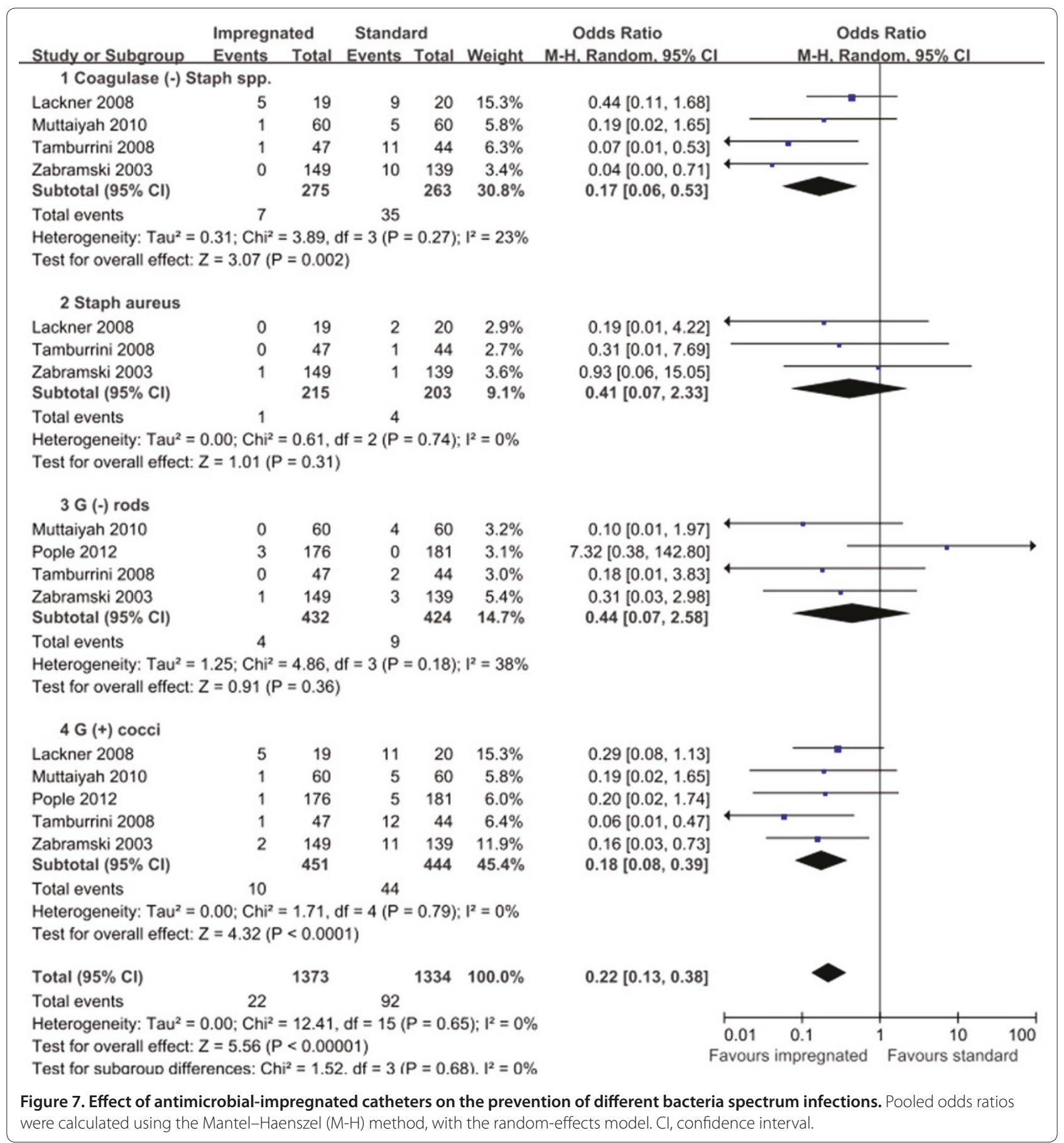

been suggested that 688 procedures are required to detect a decrease in shunt infection rate from $10 \%$ to $5 \%$ and that 438 procedures are required to detect a decrease in EVD infection rate from 15\% to 7.5\% [35]. In fact, it is difficult to obtain a sample size large enough for statistical power to find the differences. Antibiotic impregnation may increase false-negative cultures, because whether an inhibitor was used to negate the carryover effect of the antimicrobial agent into the culture medium is not often recorded [12]. Moreover, the reports of timedependent data were insufficient. We could only estimate the HR of overall infection rate indirectly from information of Kaplan-Meier curves.

The administration of PSA varied between studies, with one study administering perioperative antibiotics [27], three studies employing prolonged antibiotics until the removal of catheters $[6,10,13]$, one study not using any systemic antibiotics [7], and three studies lacking 


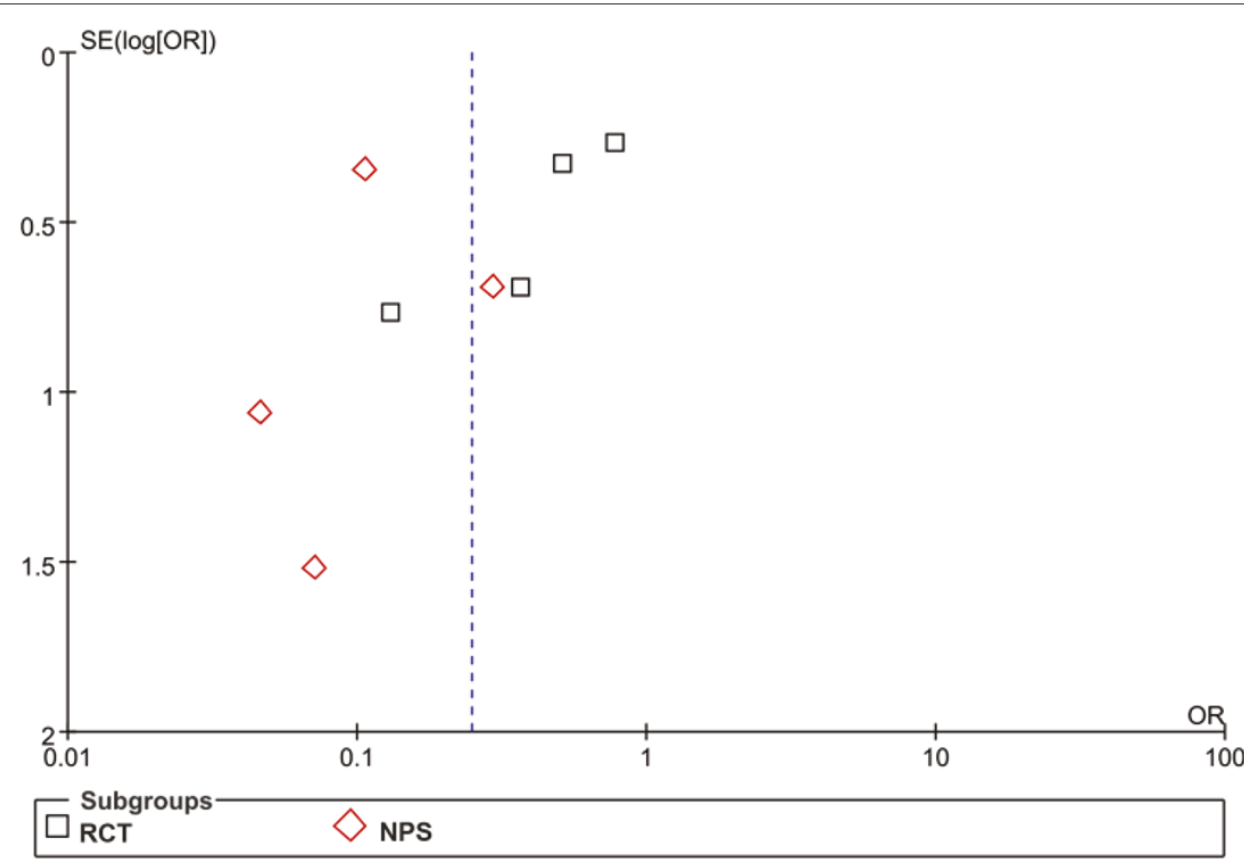

Figure 8. Funnel plot showing a small possibility of publication bias. NPS, nonrandomized prospective study; OR, odds ratio; RCT, randomized controlled trial; SE, standard error.

uniform administration or reporting no data $[8,9,12]$ (Table 1). The PSA usage may thus constitute a source of heterogeneity. Additionally, the controversial interaction between antimicrobial-impregnated catheters and PSA deserves further evaluation. Which is preferable in decreasing CFI, PSA or antimicrobial-impregnated catheters? Are the infection risks of PSA higher than catheter-impregnated antibiotics? Do combined interventions of catheter-impregnated and systemic antibiotics favor the prevention of infection? Current findings may be a dilution of the true individual effect of antimicrobial agent impregnation in the absence of any prophylactic antibiotic therapy.

Another concern is the varied criteria for diagnosing CFIs. In comparison with the Centers for Disease Control guideline, our definition of CFI demonstrated a broader profile with fewer requirements of clinical manifestations. Three studies (3/8) briefly considered positive CSF culture as infection $[6,8,10]$. Four studies $(4 / 8)$ enacted more strict criteria, requiring other abnormalities in addition to positive CSF culture, such as elevated CSF white cell counts, elevated CSF protein, or clinical manifestations $[9,12,27]$. In the absence of positive CSF cultures, we regarded elevated CSF white cell counts with clinical symptoms as evidence of infections [7]. Pople and colleagues have categorized infection outcomes into three classifications: proven infection, suspected infection, and colonization infection [13]. The various definitions not only contribute the heterogeneity, but also increase the complexity of accurately defining EVD infections. In fact, a group of five-grade criteria to describe CSF infections in ventriculostomy has been proposed, which included contamination, ventriculostomy colonization, suspected ventriculostomy-related infection, ventriculostomy-related infection, and ventriculitis [30]. Notwithstanding the difficulties in stratifying infection events, the specified classification might be helpful for future studies as demonstrated by Pople and colleagues' trial [13].

\section{Conclusion}

Our results demonstrate that antimicrobial-impregnated catheters are effective and safe in lowering the risk of CFIs and catheter colonization and are helpful in improving EVD catheter use without infection. Especially, the antimicrobial-impregnated catheters play roles against Gram-positive organisms. Antibiotic-impregnated catheters appear to be reliable options for patients requiring EVD placements. However, no sufficient evidence supports the use of silver-impregnated catheters. Further well-designed studies are needed to verify the findings of our meta-analysis.

\section{Additional file}

Additional file 1. Table presenting the Preferred Reporting Items for Systematic Reviews and Meta-analysis (PRISMA) 2009 checklist. 


\section{Abbreviations}

CFI, cerebrospinal fluid infection; Cl, confidential interval; C/R, clindamycin/ rifampin; CSF, cerebrospinal fluid; EVD, external ventricular drainage; $H R$, hazard ratio; M/R, minocycline/rifampin; NPS, nonrandomized prospective study; OR, odds ratio; PSA, prophylactic systemic antibiotics; $R C T$, randomized controlled trial.

\section{Authors' contributions}

XW performed the primary study search, extracted data, carried out statistical analysis, drafted the manuscript, and revised the manuscript. YD performed the primary study search, extracted data, carried out statistical analysis, and revised the manuscript. X-QQ carried out statistical analysis and drafted the manuscript. C-GH proposed the idea, improved the study design, drafted and revised the manuscript. L-JH improved the idea, participated in its design, drafted and revised the manuscript. Y-ML checked the study search and carried out statistical analysis. All authors read and approved the final manuscript.

\section{Competing interests}

The authors declare that they have no competing interests.

Published: 25 July 2013

\section{References}

1. Kim J-H, Desai NS, Ricci J, Stieg PE, Rosengart AJ, Hartl R, Fraser JF: Factors contributing to ventriculostomy infection. World Neurosurg 2012, 77:135-140.

2. Sonabend AM, Korenfeld Y, Crisman C, Badjatia N, Mayer SA, Connolly ES, Jr: Prevention of ventriculostomy-related infections with prophylactic antibiotics and antibiotic-coated external ventricular drains: a systematic review. Neurosurgery 2011, 68:996-1005.

3. Kubilay Z, Amini S, Fauerbach LL, Archibald L, Friedman WA, Layon AJ: Decreasing ventricular infections through the use of a ventriculostomy placement bundle: experience at a single institution. J Neurosurg 2013, 118:514-520.

4. Alleyne $\mathrm{CH}$, Jr, Hassan M, Zabramski JM: The efficacy and cost of prophylactic and perioprocedural antibiotics in patients with external ventricular drains. Neurosurgery 2000, 47:1124-1127; discussion 1127-1129.

5. Gutierrez-Gonzalez R, Boto GR: Do antibiotic-impregnated catheters prevent infection in CSF diversion procedures? Review of the literature. IInfect 2010, 61:9-20

6. Zabramski JM, Whiting D, Darouiche RO, Horner TG, Olson J, Robertson C, Hamilton AJ: Efficacy of antimicrobial-impregnated external ventricular drain catheters: a prospective, randomized, controlled trial. J Neurosurg 2003, 98:725-730.

7. Har Keong NC, Bulters DO, Richards HK, Farrington M, Sparrow OC, Pickard JD, Hutchinson PJ, Kirkpatrick PJ: The SILVER (Silver Impregnated Line Versus EVD Randomized Trial): a double-blind, prospective, randomized, controlled trial of an intervention to reduce the rate of external ventricular drain infection. Neurosurgery 2012, 71:394-404.

8. Lackner P, Beer R, Broessner G, Helbok R, Galiano K, Pleifer C, Pfausler B, Brenneis C, Huck C, Engelhardt K, Obwegeser AA, Schmutzhard E: Efficacy of silver nanoparticles-impregnated external ventricular drain catheters in patients with acute occlusive hydrocephalus. Neurocrit Care 2008, 8:360-365.

9. Muttaiyah S, Ritchie S, John S, Mee E, Roberts S: Efficacy of antibioticimpregnated external ventricular drain catheters. J Clin Neurosci 2010 17:296-298.

10. Tamburrini G, Massimi L, Caldarelli M, Di Rocco C: Antibiotic impregnated external ventricular drainage and third ventriculostomy in the management of hydrocephalus associated with posterior cranial fossa tumours. Acta Neurochir (Wien) 2008, 150:1049-1055; discussion 1055-1056.

11. Wong GKC, Poon WS, Ng SCP, Ip M: The impact of ventricular catheter impregnated with antimicrobial agents on infections in patients with ventricular catheter: interim report. Acta Neurochir Supp/ 2008, 102:53-55.

12. Wong GKC, Ip M, Poon WS, Mak CWK, Ng RYT: Antibiotics-impregnated ventricular catheter versus systemic antibiotics for prevention of nosocomial CSF and non-CSF infections: a prospective randomised clinical trial. J Neurol Neurosurg Psychiatry 2010, 81:1064-1067.

13. Pople I, Poon W, Assaker R, Mathieu D, lantosca M, Wang E, Zhang LW, Leung G, Chumas P, Menei P, Beydon L, Hamilton M, Kamaly I, Lewis S, Ning W, Megerian JT, McGirt MJ, Murphy JA, Michael A, Meling T: Comparison of infection rate with the use of antibiotic-impregnated vs standard extraventricular drainage devices: a prospective, randomized controlled trial. Neurosurgery 2012, 71:6-13.

14. Arabi Y, Memish ZA, Balkhy HH, Francis C, Ferayan A, Al Shimemeri A, Almuneef MA: Ventriculostomy-associated infections: incidence and risk factors. Am J Infect Control 2005, 33:137-143.

15. Lyke KE, Obasanjo OO, Williams MA, O'Brien M, Chotani R, Perl TM: Ventriculitis complicating use of intraventricular catheters in adult neurosurgical patients. Clin Infect Dis 2001, 33:2028-2033.

16. Moher D, Liberati A, Tetzlaff J, Altman DG: Preferred reporting items for systematic reviews and meta-analyses: the PRISMA statement. PLoS Med 2009, 6:e1000097.

17. Horan TC, Andrus M, MA D: CDC/NHSN surveillance definition of healthcare-associated infection and criteria for specific types of infections in the acute care setting. Am J Infect Control 2008, 36:309-322.

18. Jadad AR, Moore RA, Carroll D, Jenkinson C, Reynolds DJ, Gavaghan DJ, McQuay HJ: Assessing the quality of reports of randomized clinical trials: is blinding necessary? Control Clin Trials 1996, 17:1-12.

19. Wells GA, Shea B, O'Connell D, Peterson J, Welch V: The Newcastle-Ottawa Scale (NOS) for Assessing the Quality of Nonrandomised Studies in Metaanalyses. [http://www.ohri.ca/programs/clinical_epidemiology/oxford.asp]

20. Bradburn MJ, Deeks JJ, Berlin JA, Russell Localio A: Much ado about nothing: a comparison of the performance of meta-analytical methods with rare events. Stat Med 2007, 26:53-77.

21. Ades AE, Lu G, Higgins JP: The interpretation of random-effects metaanalysis in decision models. Med Decis Making 2005, 25:646-654

22. Sweeting MJ, Sutton AJ, Lambert PC: What to add to nothing? Use and avoidance of continuity corrections in meta-analysis of sparse data. Stat Med 2004, 23:1351-1375.

23. Tierney JF, Stewart LA, Ghersi D, Burdett S, Sydes MR: Practical methods for incorporating summary time-to-event data into meta-analysis. Trials 2007 8:16.

24. Engauge Digitizer Version 2.15 [http://digitizer.sourceforge.net]

25. Higgins JPT, Green S, Collaboration C: Cochrane Handbook for Systematic Reviews of Interventions. Chichester, UK: Wiley Online Library; 2008.

26. Egger M, Smith GD, Altman D: Systematic Reviews in Health Care: Meta-analysis in Context. London: BMJ Books; 2008

27. Harrop JS, Sharan AD, Ratliff J, Prasad S, Jabbour P, Evans JJ, Veznedaroglu E, Andrews DW, Maltenfort M, Liebman K, Flomenberg P, Sell B, Baranoski AS, Fonshell C, Reiter D, Rosenwasser RH: Impact of a standardized protocol and antibiotic-impregnated catheters on ventriculostomy infection rates in cerebrovascular patients. Neurosurgery 2010, 67:187-191; discussion 191.

28. Gilbert RE, Harden M: Effectiveness of impregnated central venous catheters for catheter related blood stream infection: a systematic review. Curr Opin Infect Dis 2008, 21:235-245.

29. Abla AA, Zabramski JM, Jahnke HK, Fusco D, Nakaji P: Comparison of two antibiotic-impregnated ventricular catheters: a prospective sequential series trial. Neurosurgery 2011, 68:437-442; discussion 442.

30. Lozier AP, Sciacca RR, Romagnoli MF, Connolly ES, Jr: Ventriculostomy-related infections: a critical review of the literature. Neurosurgery 2002, 51:170-181; discussion 181-182.

31. Ritz R, Roser F, Morgalla M, Dietz K, Tatagiba M, Will BE: Do antibioticimpregnated shunts in hydrocephalus therapy reduce the risk of infection? An observational study in 258 patients. BMC Infect Dis 2007, 7:38

32. Ratilal B, Costa J, Sampaio C: Antibiotic prophylaxis for surgical introduction of intracranial ventricular shunts. Cochrane Database Syst Rev 2006, 3:CD005365.

33. Thomas R, Lee S, Patole S, Rao S: Antibiotic-impregnated catheters for the prevention of CSF shunt infections: a systematic review and meta-analysis. Br J Neurosurg 2012, 26:175-184.

34. Baker SG, Lindeman KS: Rethinking historical controls. Biostatistics 2001, 2:383-396

35. Gutierrez-Gonzalez R, Boto GR: Do antibiotic-impregnated catheters prevent infection in CSF diversion procedures? Review of the literature. $J$ Infect 2010, 61:9-20.

doi:10.1186/cc12608

Cite this article as: Wang $X$, et al:: Clinical review: Efficacy of antimicrobialimpregnated catheters in external ventricular drainage - a systematic review and meta-analysis. Critical Care 2013, 17:234. 